\title{
Epidemiological, Diagnostic and Therapeutic Aspects of Cardiogenic Shock in Children at the Albert Royer Children's Hospital in Dakar
}

\author{
Mohameth Mbodj1 ${ }^{*}$, Amadou Lamine Fall1,2, Aliou Thiongane ${ }^{1,2}$, Ibrahima Diagne ${ }^{3,4}$, \\ Amadou Sow ${ }^{1,5}$, Serigne T. Ndiaye1, Khadim Bop ${ }^{1}$, Papa S. Sow ${ }^{1}$, Ousmane Ndiaye ${ }^{1,2}$ \\ ${ }^{1}$ Albert Royer National Children Hospital, Dakar, Senegal \\ ${ }^{2}$ Cheikh Anta Diop University, Dakar, Senegal \\ ${ }^{3}$ Gaston Berger University, Saint Louis, Senegal \\ ${ }^{4}$ Saint Louis Regional Hospital, Saint Louis, Senegal \\ ${ }^{5}$ Abass Ndao Hospital Center, Dakar, Senegal \\ Email: *mohamethmbodj@gmail.com
}

How to cite this paper: Mbodj, M., Fall, A.L., Thiongane, A., Diagne, I., Sow, A., Ndiaye, S.T., Bop, K., Sow, P.S. and Ndiaye, O. (2021) Epidemiological, Diagnostic and Therapeutic Aspects of Cardiogenic Shock in Children at the Albert Royer Children's Hospital in Dakar. Open Journal of Pediatrics, 11, 669-675.

https://doi.org/10.4236/ojped.2021.114062

Received: November 1, 2021

Accepted: December 5, 2021

Published: December 8, 2021

Copyright $\odot 2021$ by author(s) and Scientific Research Publishing Inc. This work is licensed under the Creative Commons Attribution International License (CC BY 4.0).

http://creativecommons.org/licenses/by/4.0/

\section{(c) (i) Open Access}

\begin{abstract}
Introduction: The objective of this study was to evaluate the epidemiological, diagnostic and therapeutic aspects of cardiogenic shock in children at the Albert Royer Children's Hospital in Dakar. Methods: This was a retrospective, descriptive and analytical study from January 2020 to February 2021, including all children aged 2 months to 16 years hospitalised for cardiogenic shock diagnosed on the basis of clinical and ultrasound criteria. Results: During the study, 38 patients were hospitalised for cardiogenic shock. The hospital prevalence was $4.2 \%$. The mean age of onset of shock was 64 months, and there was a predominance of females with a sex ratio of 1.92. Consanguinity was found in $42 \%$ of the patients. Consanguinity was found in $42 \%$ of patients. Infection was identified as a trigger for cardiogenic shock in 18 (52.9\%) of our patients. The most common type of heart disease was rheumatic heart disease in $12(32 \%)$ of the patients. The mortality rate was $65.8 \%$. Conclusion: Cardiogenic shock is a diagnostic and therapeutic emergency. Its prevalence and mortality are still high in developing countries.
\end{abstract}

\section{Keywords}

Heart Disease, Consanguinity, CMDH, Shortening Fraction, Senegal

\section{Introduction}

Cardiogenic shock is an acute circulatory failure due to impaired myocardial 
contractility resulting in decreased cardiac output and pulmonary congestion [1]. It is an immediate life-threatening emergency. More frequent in adults, in whom it represents the main cause of mortality of patients hospitalised for myocardial infarction, cardiogenic shock is much rarer in children [2]. In children and infants, it can occur de novo in a healthy heart or in pre-existing congenital or chronic heart disease [3]. Heart failure in paediatric populations is a major public health problem. It is associated with high rates of hospitalisation, disability and mortality in high-income countries, but its burden is poorly documented in developing countries [4]. There are few published studies on cardiogenic shock in children worldwide and none in Senegal. It is in this context that we conducted this study to evaluate the epidemiological, diagnostic and therapeutic aspects of cardiogenic shock in children.

\section{Methodology}

This work was carried out at the Centre Hospitalier National d'Enfants Albert Royer (CHNEAR), which is the only exclusively paediatric level 3 public health establishment (EPS) in the Dakar region. It houses a paediatric cardiology department where the majority of children with congenital or acquired heart disease are followed. The study was retrospective, descriptive and analytical over a period of 14 months (from 01 January 2020 to 28 February 2021). Epidemiological, diagnostic, therapeutic and evolutionary parameters were collected from medical records and hospital registers. We included all children aged 2 months to 16 years hospitalised for cardiogenic shock confirmed on the basis of clinical criteria such as: tachycardia, prolonged skin recolouration time, arterial hypotension associated with signs of heart failure (hepatomegaly, gallop rhythm, cardiomegaly, oedema of the lower limbs) and echographic criteria: alteration of the RF (shortening fraction). The data were entered in Excel and analysed using SPSS (Statistical Package for Social Sciences) Statistics version 25. The significance threshold was retained for a p-value $<0.05$. Patients were initially hospitalised in the reception and emergency department for stabilisation before being transferred to the paediatric cardiology department for follow-up. The duration of follow-up will depend on the patient's progress and the type of heart disease.

\section{Results}

During the period of our study 894 patients were hospitalised, of whom 164 for heart disease and 38 for cardiogenic shock, i.e. a hospital prevalence of $4.2 \%$. Cardiogenic shock occurred in $23 \%$ of patients hospitalised for heart disease. The mean age was 64 months with a standard deviation of 67, the sex ratio was 1.92 in favour of girls. Parental consanguinity was found in 16 (42\%) of our patients. The socio-economic level of our patients was considered low in 28 patients (74\%), 26 (68\%) of the patients came from the suburbs of Dakar. The socio-demographic characteristics are summarised in Table 1. Heart disease was diagnosed before the onset of cardiogenic shock in 24 (89\%) patients. Infection 
Table 1. Socio-demographic characteristics.

\begin{tabular}{clc}
\hline Parameters & & Percentage \% \\
\hline Sex & Feminine & 66 \\
Age & Masculine & 34 \\
& $0-23$ months & 55 \\
& $24-59$ months & 5 \\
& $60-120$ months & 11 \\
Socio-economic level & More than 120 months & 29 \\
& Medium & 74 \\
& Suburbs of Dakar & 26 \\
& Dakar & 68 \\
& Outside the Dakar region & 14 \\
& & 18 \\
\hline
\end{tabular}

Table 2. Distribution according to the triggering factor of cardiogenic shock.

\begin{tabular}{ccc}
\hline Triggering factor & Staff & Percentage (\%) \\
\hline Infection & 18 & 52.9 \\
Therapeutic rupture & 8 & 23.6 \\
Anaemia & 2 & 5.9 \\
Not found & 6 & 17.6 \\
Total & 34 & 100 \\
\hline
\end{tabular}

was identified as a trigger for cardiogenic shock in 18 (52.9\%) of our patients (Table 2). The most common type of heart disease was rheumatic heart disease in $12(32 \%)$ of the patients (Figure 1). The most common clinical signs were tachycardia, which was present in all our patients, as well as dyspnoea and cold extremities (Table 3). Respiratory distress was severe in 31 patients (81.6\%) according to the clinical classification (CRS). All our patients had received dobutamine and oxygen therapy, 36 patients had received furosemide, 16 (42\%) were intubated and ventilated. The evolution was marked by death in 25 of our patients; that is $65.8 \%$ (Table 4). All our deceased patients had respiratory distress classified as moderate or severe according to the CRS. All patients with a shock duration of more than 12 hours died (Table 5). All patients admitted with a shortening fraction (RF) on cardiac ultrasound of less than 16 (44\%) died. Patients admitted with a RF of more than 22 had a favourable outcome.

\section{Discussion}

During the study period, 894 patients were hospitalised at the CHNEAR reception and emergency department, including 164 for heart disease and 38 for cardiogenic shock, i.e. a hospital prevalence of $4.2 \%$. This prevalence is comparable 


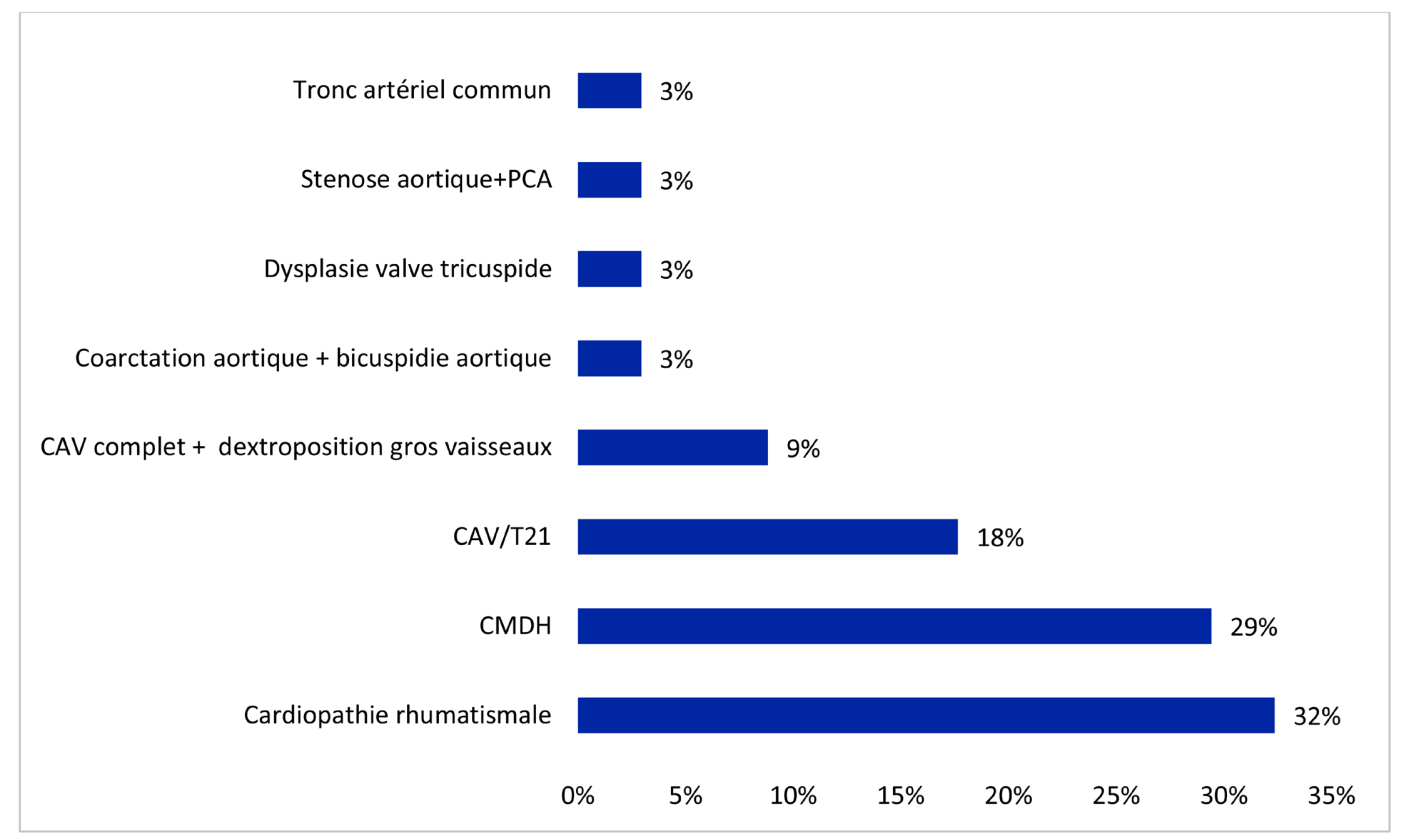

CMDH: Hypokinetic Dilated Cardiomyopathy; PCA: Persistence of the Arterial Canal; T21: Down syndrome.

Figure 1. Type of heart disease found.

Table 3. Clinical signs of cardiogenic shock.

\begin{tabular}{ccc}
\hline Signs & Staff & Percentage (\%) \\
\hline Hepatomegaly & 38 & 100 \\
Turgor of jugular veins & 24 & 63 \\
Chinstrap hepatic reflux & 34 & 89 \\
Edema of the lower limbs & 13 & 34 \\
Dyspnoea & 38 & 100 \\
Cold ends & 34 & 89 \\
Profuse sweats & 9 & 23 \\
Skin recoloration time & 38 & 100 \\
Tachycardia & 38 & 100 \\
\hline
\end{tabular}

Table 4. Patient progress.

\begin{tabular}{ccc}
\hline Evolution & Staff & Percentage (\%) \\
\hline Favorable & 13 & 34.2 \\
Death & 25 & 65.8 \\
Total & 38 & 100.0 \\
\hline
\end{tabular}

Table 5. Evolution according to the duration of the shock.

\begin{tabular}{|c|c|c|c|c|}
\hline & & \multicolumn{2}{|c|}{ Evolution } & \multirow{2}{*}{ Total } \\
\hline & & Unfavourable & Favorable & \\
\hline \multirow{2}{*}{ Duration (hours) } & $\leq 12 \mathrm{~h}$ & 18 & 13 & 31 \\
\hline & $13 \mathrm{~h}+$ & 7 & 0 & 7 \\
\hline \multicolumn{2}{|c|}{ Total } & 25 & 13 & 38 \\
\hline
\end{tabular}


to that found by Abrik et al. in Morocco in 2017 (3.7\%) [5]. The higher prevalence in our study could be explained by the lack of paediatric cardiologists in the periphery. Most of our patients, $31 \%$ or $81 \%$, came from the Dakar region. These results are comparable to the results of Sylla et all in 2017 [6]. Dakar is the location of the CHNEAR which is a referral centre that polarises the population of Dakar and its suburbs. The socio-economic level was low in $73 \%$ of our patients. Azagoh-Kouadio et al. [7] found 61\% of low socio-economic level in Côte d'Ivoire. Our results are in line with the literature which identifies low socio-economic level as a risk factor for the occurrence of acquired heart disease [8] [9]. Cardiogenic shock in children occurs most often in the presence of known heart disease [1] [10]. Indeed, in our series, $89 \%$ of the patients already had a known heart disease. However, we noted that $11 \%$ of our patients had no known cardiac disease. This could be explained by the number of under-diagnosed heart diseases linked to the lack of cardio pediatricians in Senegal. In our series, the etiologies found are similar to the data found in the literature. Indeed, Paut et al. in 2010, found 30\% of congenital heart disease in the etiologies of cardiogenic shock [11] [12]. Furthermore, Chebab et al. in 2005 found a predominance of rhythm disorders and coronary birth defects (ALCAPA) in the etiologies of cardiogenic shock in children. These aetiologies were not found in our series and this could be due to an under-diagnosis because of the lack of exploration means such as the paediatric ECG. Rhythm disorders and coronary birth defects have been found in several European series and constitute the main etiology of cardiogenic shock in children [13] [14]. Blood pressure was unstoppable in 39\% of our patients. However, Randrianarison et al. in 2013 found unstoppable blood pressure in $22 \%$ of patients [15]. The higher rate of impregnable blood pressure in the study could be explained by the fact that the majority of our patients came from the periphery, which delayed management. However, the recognition of cardiogenic shock is a diagnostic and above all a therapeutic emergency, as any delay in initiating appropriate treatment may lead to irreversible visceral damage. The presence of arterial hypotension is a sign of the advanced stage of cardiogenic shock in children [5].

The main therapeutic means used in our study were dobutamine and noradrenaline. In addition, 16 of our patients received mechanical ventilation. Other molecules are increasingly used in the treatment of cardiogenic shock, notably phosphodiesterase III (PDE III) inhibitors such as milrinone [16]. None of our patients benefited from ECMO (Extracorporeal Membrane Oxygenation) which is a therapeutic means indicated in refractory cardiogenic shock [17] [18]. The death rate in our series was $65 \%$. The higher mortality in our study could be explained by several factors. Indeed, in our study, several therapeutic means were not used, notably second-line means in case of refractory shock, and the delay in the management of patients coming from the periphery. This can be explained by the technical platform which is still at a low level in our developing countries. Several means of resuscitation are not available in our hospitals. 
The evolution of the patients depended on the severity' of the cardiac damage. Indeed, in our study, all the patients who had an RF below 16 died and those with an RF above 22 evolved well. In several scientific series, the RF was considered as a prognostic element. Indeed, Bounhoure et al. in 2002 demonstrated that the lower the RF, the higher the risk of death [19].

\section{Conclusion}

Cardiogenic shock is a diagnostic and therapeutic emergency. It is a rare pathology in the pediatrics setting with little data available, especially in developing countries. Its diagnosis is often late in our country and its mortality is still very high. The improvement of the mortality rate could be achieved by improving the technical platform and the training of health personnel.

\section{Conflicts of Interest}

The authors declare no conflicts of interest regarding the publication of this paper.

\section{References}

[1] Brissaud, O., Thambo, J.B., Guichoux, J., Nolent, P. and Dauger, S. (2013) Cardiogenic Shock in Children. In: Paut, O. and Orliaguet, G., Eds., Réanimation Pédiatrique, Springer, Paris, 77-107. https://doi.org/10.1007/978-2-8178-0407-1_4

[2] Cecconi, M., De Backer, D., Antonelli, M., Beale, R., Bakker, J., Hofer, C., et al. (2014) Consensus on Circulatory Shock and Hemodynamic Monitoring. Task Force of the European Society of Intensive Care Medicine. Intensive Care Medicine, 40, 1795-1815. https://doi.org/10.1007/s00134-014-3525-Z

[3] Kamel, D.E.I. (2019) Interest of Hemodynamic Monitoring in the Management of Acute Circulatory Failure (ACF) in Children in Intensive Care. Faculty of Medicine of Oran, Oran.

[4] Sibetcheu, A.T., Agbor, V.N., Nyaga, U.F., Bigna, J.J. and Noubiap, J.J. (2018) Epidemiology of Heart Failure in Pediatric Populations in Low- and Middle-Income Countries: A Protocol for a Systematic Review. Systematic Reviews, 7, Article No. 52. https://doi.org/10.1186/s13643-018-0717-6

[5] Abrik, A. (2017) Cardiogenic Shock: Epidemiology and Ethiopathogenesis. Sidi Mouhamed Ben Abdellah University, Morocco.

[6] Sylla, P.M. (2017) Hypokinetic Dilated Cardiomyopathy in Children. Dakar Faculty of Medicine, UCAD, Senegal.

[7] Azagoh-Kouadio, R., Diby, K.F., Yeboua, K.R., Avi, C., Aka-Tanoh, A.H., Gnaba, L.A., et al. (2019) Acquired Heart Disease in Children in Côte d'Ivoire: A Retrospective Multicentric Study. International Journal of Medical Sciences: Abj-RISM, 4 , 9.

[8] Fall, A.L., Sow, A., Basse, I., Coundoul, A.M., Monoue, F.P.S., Thiongane, A., et al. (2020) Congenital Cyanogenic Heart Disease in Children: About 420 Cases in Africa. World Journal of Cardiovascular Surgery, 10, 83-90. https://doi.org/10.4236/wjcs.2020.106011

[9] Diby, K.F., Adoubi, K.A., Gnaba, L.A., Aka-Tanoh, K.A.-H., Ayegnon, K.G. and Asse, K.V. (2019) Congenital Heart Disease at the University Hospital of Bouaké. 
Clinical and Evolutionary Aspects. International Journal of Medical Sciences. Abj-RISM, 2, 7.

[10] Renolleau, S., Rambaud, J. and Durandy, A. (2014) Acute Cardiac Insuffisance in Children. Pediatric Realities, 4.

[11] Paut, O., Ughetto, F. and Labenne, M. (2010) Pediatric Particularities of Shock. SFAR, 50 .

[12] Chehab, G., Saliba, Z., Chedid, P., Gerbaka, B. and Mourani, C. (2005) Cardiogenic Shock Revealing Heart Disease in Infancy and Childhood: A Lebanese Multicentric Study. Journal Medical Libanais, 53, 202-207.

[13] Beggs, S., Thompson, A., Nash, R., Tompson, A. and Peterson, G. (2009) Cardiac Failure in Children. Expert Committee on the Selection and Use of Essential Medicines, 31 .

[14] di Bernardo, S., Boulos, T., Mivelaz, Y. and Sekarski, N. (2011) Heart Failure in Children, State of Knowledge, Treatment Perspectives. Pediatrica, 22, 4.

[15] Randrianarison, L.S. (2013) Cardiogenic Shock at the USIC Befelatanana: Epidemiological and Clinical Aspects, Evolution and Management. Universite d'Antananarivo, Antananarivo.

[16] Léopold, V. and Gayat, E. (2019) New Developments in the Pharmacological Treatment of Cardiogenic Shock. Le praticien en anesthésie réanimation, 23, 19-24.

https://doi.org/10.1016/j.pratan.2018.12.002

[17] Amodeo, I., Di Nardo, M., Raffaeli, G., Kamel, S., Macchini, F., Amodeo, A., et al. (2021) Neonatal Respiratory and Cardiac ECMO in Europe. European Journal of Pediatrics, 180, 1675-1692. https://doi.org/10.1007/s00431-020-03898-9

[18] Brissaud, O., Botte, A., Cambonie, G., Dauger, S., de Saint Blanquat, L., Durand, P., et al. (2016) Experts' Recommendations for the Management of Cardiogenic Shock in Children. Annals of Intensive Care, 6, Article No. 14. https://doi.org/10.1186/s13613-016-0111-2

[19] Bounhoure, J.-P., Massabuau, P., Galinier, M., Jordan, C., Laurent, J.-P. and Marco, J. (2002) Heart Failure with Preserved Systolic Ventricular Function: Clinical, Echocardiographic and Evolutionary Aspects. Prognostic Factors. Bulletin de L'Académie Nationale de Médecine, 186, 1003-1014. https://doi.org/10.1016/S0001-4079(19)34245-1 